PROCEEDINGS OF THE AMERICAN MATHEMATICAL SOCIETY

Volume 124, Number 5, May 1996

\title{
NON-SIMPLE LINKS WITH TUNNEL NUMBER ONE
}

\author{
MARIO EUDAVE-MUÑOZ AND YOSHIAKI UCHIDA
}

(Communicated by Ronald J. Stern)

\begin{abstract}
We determine all non-simple links which admit an unknotting tunnel, i.e. links which contain an essential annulus or torus in its exterior and have tunnel number one.
\end{abstract}

A link $l$ is non-simple if there is an essential annulus or torus properly embedded in the exterior of $l, E(l)$, that is, there is an annulus or torus which is incompressible, $\partial$-incompressible, and non $\partial$-parallel. A link $l$ admits an unknotting tunnel if there is a properly embedded $\operatorname{arc} \tau$ in $E(l)$ such that $E(l \cup \tau)$ is a handlebody; in that case we say that $l$ has tunnel number one. If $l$ is not a knot and admits an unknotting tunnel, then necessarily $l$ has two components, say $l=l_{1} \cup l_{2}$, and any unknotting tunnel for $l$ connects $l_{1}$ to $l_{2}$.

In this paper we give a complete list of all non-simple links which admit an unknotting tunnel. Knots admitting unknotting tunnels are classified in [MS], [E]. First we show that if $l$ is a non-simple link which has an unknotting tunnel $\tau$, then there is an essential annulus $A$ in $E(l)$ disjoint from $\tau$. This divides the non-simple links into two types, depending if $\partial A=a_{1} \cup a_{2}$ lie on different, or on the same component of $\partial E(l)$. In Theorem 1 (2) we give a list of the non-simple links having an annulus as in the first (second) case.

Lemma. Let $l$ be a non-simple link with an unknotting tunnel $\tau$. Then there exist an essential annulus $A$ in $E(l)$ such that $A \cap \tau=\varnothing$.

Proof. Let $M=E(l \cup \tau)$. $M$ is a genus 2-handlebody. $E(l)$ is obtained by adding a 2-handle to $M$ along a curve $\alpha$ on $\partial M$ which is a cocore of the tunnel $\tau$. Clearly $\partial M$ is compressible, $\partial M \backslash \alpha$ is incompressible, and $\alpha$ is a separating curve on $\partial M$. If there is an essential annulus or torus in $E(l)$, then by Theorems 1 and 2 of [E], there is an essential (incompressible, not parallel to a surface in $\partial M$ ) annulus $A$ in $M$ disjoint from $\alpha$, that is, an essential annulus in $E(l)$ disjoint from $\tau$.

Theorem 1. Let $l$ be a non-simple link with unknotting tunnel $\tau$, and $A$ an essential annulus disjoint from $\tau$. Suppose that $\partial A=a_{1} \cup a_{2}$ lie on different components of $\partial E(l)$. Then one of the following cases occurs:

Received by the editors May 5, 1994

1991 Mathematics Subject Classification. Primary 57M25.

Key words and phrases. Essential annulus or torus, non-simple link, unknotting tunnel.

The first author is grateful to the Osaka City University for its hospitality during the winter of 1993, where part of this work was done. The second author was partially supported by Grantin-aid for Scientific Research, Ministry of Education, Science and Culture: A fellow of the Japan Society for the Promotion of Science for Japanese Junior Scientists.

(C)1996 American Mathematical Society 


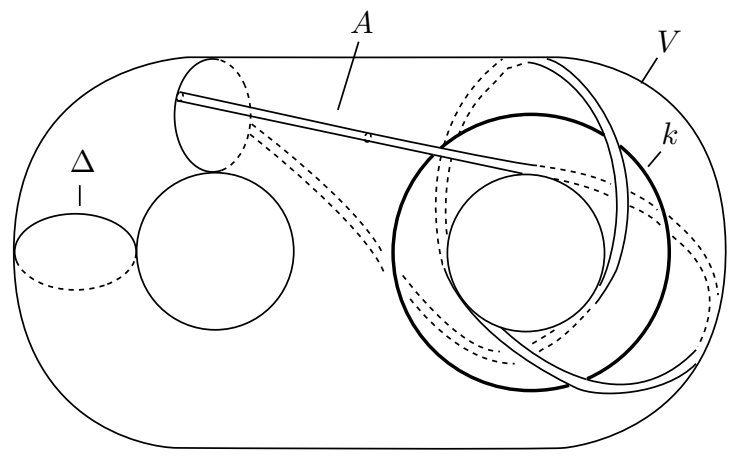

Figure 1

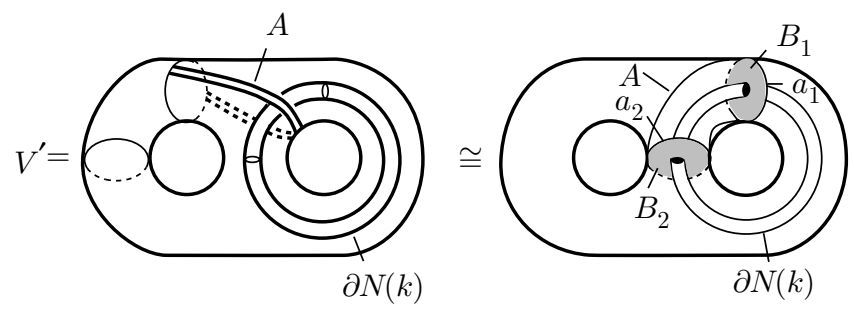

FigURE 2

(i) If $a_{1}$ is a meridian of $N\left(l_{1}\right)$ and $a_{2}$ is (necessarily) isotopic to $l_{2}$ in $N\left(l_{2}\right)$, then $l$ is a connected sum of a two-bridge knot $k$ and the Hopf link.

(ii) If $a_{1}$ is isotopic to $l_{1}^{n}(n \geq 2)$ in $N\left(l_{1}\right)$ and $a_{2}$ is isotopic to $l_{2}$ in $N\left(l_{2}\right)$, then $l_{2}$ is a torus knot and $l_{1}$ is its core.

(iii) If $a_{i}$ is isotopic to $l_{i}$ in $N\left(l_{i}\right)(i=1,2)$, then $l$ is a torus link with type $(2,2 p)$, $p \in \mathbb{N}$.

(iv) If $a_{i}$ is isotopic to $l_{i}^{n_{i}}\left(n_{i} \geq 2\right)$ in $N\left(l_{i}\right)(i=1,2)$, then $l$ is the Hopf link.

Proof. By the definition of a tunnel, $E(l \cup \tau)$ is a genus two handlebody $V$. Since each component of $\partial A$ lies on a different component of $\partial E(l)$ and $A$ is essential in $E(l), A$ is a non-separating essential annulus in $V$. According to [K, Lemma 3.2 (ii)], we can assume that the pair $(V, A)$ is like in Figure 1.

Note that there exists a meridian disk $\Delta$ of $V$ such that $\Delta \cap A=\varnothing$. Now, we consider the knot $k$ in $V$ indicated in Figure 1. Then $V^{\prime}=V \backslash N^{\circ}(k)$ is as shown in Figure 2.

Let $\alpha$ be a simple closed curve on $\partial V$ which is a cocore of the tunnel $\tau$. Since each component of $\partial A$ lie on a different component of $E(l), \alpha$ separates $a_{1}$ from $a_{2}$ on $\partial V$. Let $B_{1}, B_{2}$ be the annuli indicated in Figure 2. Then $\left\{B_{1}, B_{2}\right\}$ cuts $V^{\prime}$ into a genus two handlebody $V^{\prime \prime}$. Let $a_{i}^{\prime}, a_{i}^{\prime \prime}$ be copies of $a_{i}(i=1,2)$ on $\partial V^{\prime \prime}$. (See Figure 3.)

We can consider $\left(V^{\prime \prime}, \partial N(k)\right)$ as a trivial tangle. Since the curve $\alpha$ separates $\left\{a_{1}^{\prime}, a_{1}^{\prime \prime}\right\}$ from $\left\{a_{2}^{\prime}, a_{2}^{\prime \prime}\right\}$, it follows that $\alpha$ is as shown in Figure 4(i). Then, we can assume that $V^{\prime}$ is as shown in Figure 4(ii). By attaching a 2-handle to $V^{\prime}$ along $\alpha$, we have $\Sigma=E(l) \backslash N^{\circ}(k)$. Now, after moving $\partial N\left(l_{2}\right)$ through $k$ along the annulus 


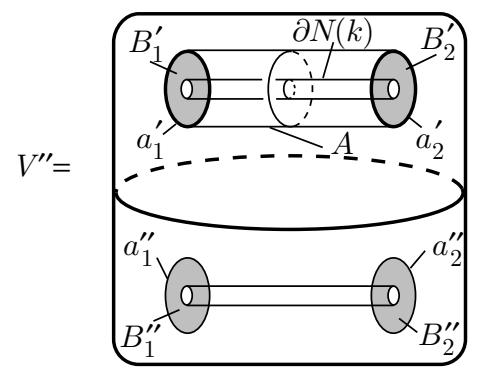

Figure 3

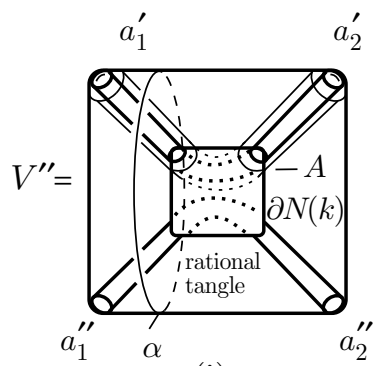

(i)

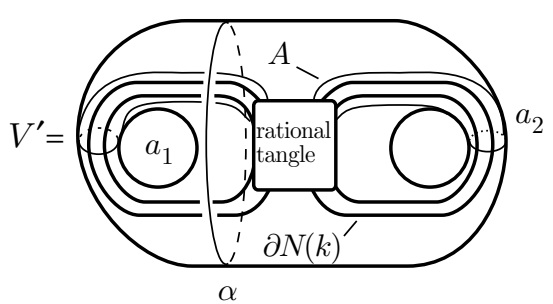

(ii)

FiguRE 4

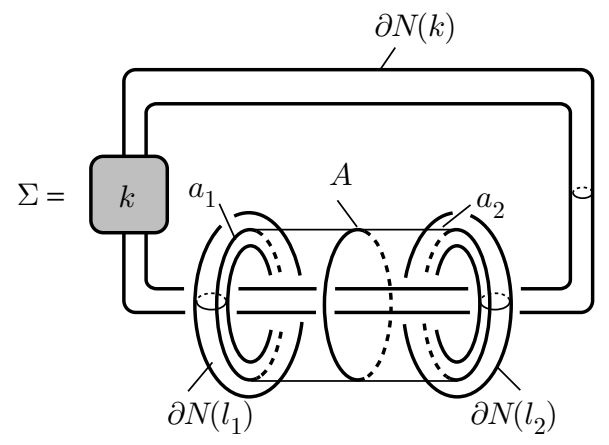

FiguRE 5

$A, \Sigma$ looks like in Figure 5. $\partial N(k)$ as in Figure 5 determines the exterior of a knot $K$ in $S^{3}$. Note that $K$ is a two-bridge knot.

Case (i). Since $a_{2}$ is isotopic to $l_{2}$ in $N\left(l_{2}\right), \Sigma^{\prime}=\Sigma \cup N\left(l_{2}\right)$ is indicated in Figure 6(i).

We can exchange $\partial N\left(l_{1}\right)$ for $\partial N(k)$ by a homeomorphism of $\Sigma$. (See Figure 6(ii). If we cut $\Sigma^{\prime}$ along the annulus $C$ shown in Figure 6 , it is easy to find the homeomorphism. It can also be seen applying the bulb trick, where $\partial N\left(l_{1}\right)$ becomes knotted while $\partial N(k)$ is being unknotted.) But because $a_{1}$ is a meridian of $N\left(l_{1}\right)$, it follows that $\left(S^{3}, l\right)$ is as shown in Figure 6(iii), by a surgery description. The manifold is $S^{3}$, so $s / r= \pm 1 / n, n \in \mathbb{N}$. 


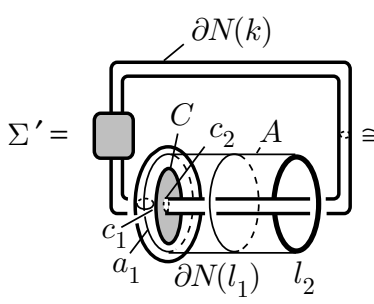

(i)

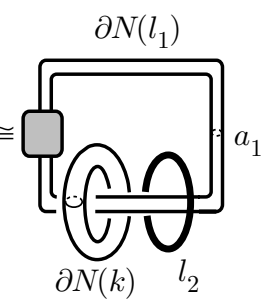

(ii)

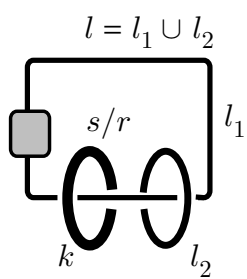

(iii)

FiguRE 6

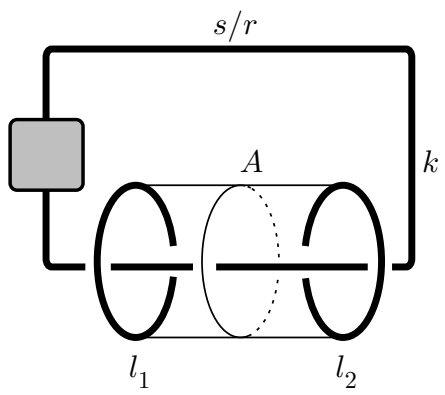

Figure 7

This completes the proof of Case (i).

Case (ii). Since $a_{2}$ is isotopic to $l_{2}$ in $N\left(l_{2}\right), \Sigma^{\prime}=\Sigma \cup N\left(l_{2}\right)$ is indicated in Figure 6(i). Let $X=N\left(l_{1}\right) \cup N(C) \cup N(k), c_{1}=N\left(l_{1}\right) \cap C, c_{2}=N(k) \cap C$. Then $S^{3}=X \cup E(X), E(X)$ is homeomorphic to $E(K)$, and $a_{1}$ is a meridian of $E(K)$. Now, we consider the following two cases:

(a) $K$ is a non-trivial two-bridge knot, or

(b) $K$ is a trivial knot.

Case (ii-a). $X$ must be a solid torus, for otherwise $X \cup E(X)$ could not be $S^{3}$. Since $a_{1} \cap C=\varnothing, a_{1}$ is a curve on $\partial X$. Then $a_{1}$ is not a meridian of $X$, because $a_{1}$ is isotopic to $l_{1}^{n}(n \geq 2)$ in $N\left(l_{1}\right)$. Now $S^{3}=X \cup_{a_{1}} E(X)$. This is a contradiction, because every non-trivial two-bridge knot has Property P by [T]. Hence Case (ii-a) does not occur.

Case (ii-b). We can consider $X$ as a union of two solid tori attached along an annulus. So $X$ must be a torus knot exterior, and $l_{1}$ is its core. Since $K$ is trivial, $E(X)(\cong E(K))$ is a solid torus and $l_{2}$ is its core. Then, we have that $l_{2}$ is a torus knot and $l_{1}$ is its core. This completes the proof of Case (ii).

Case (iii). Since $a_{i}$ is isotopic to $l_{i}$ in $\left(N_{i}\right)(i=1,2),\left(S^{3}, l_{1} \cup l_{2}\right)$ is indicated in Figure 7 by a surgery description, where $s / r$ is a surgery coefficient.

Since $A$ is incompressible, this Dehn surgery on $K$ must be non-trivial. Every non-trivial two-bridge knot has Property $\mathrm{P}$ by [T], so $K$ must be trivial, and $r / s$ must be $\pm 1 / n$. Then $l$ is a torus link, with type $(2,2 n), n \in \mathbb{N}$.

This completes the proof of Case (iii).

Case (iv). Let $Y=N\left(l_{1}\right) \cup N(A) \cup N\left(l_{2}\right)$. Then $S^{3}=Y \cup E(Y)$. Since $a_{i}$ is isotopic to $l_{1}^{n}(n \geq 2), Y$ must be a non-trivial torus knot exterior and $E(Y)$ must 
be a solid torus. Since $l_{1}$ (resp. $l_{2}$ ) is the core of $N\left(l_{1}\right)$ (resp. $N\left(l_{2}\right)$ ), $l$ is the Hopf link.

This completes the proof of Case (iv).

Note that any link satisfying one of Cases (i)-(iv) of Theorem 1 is non-simple and has an unknotting tunnel.

We describe two classes of non-simple links which admit unknotting tunnels.

(1) Let $R$ be a standard solid torus in $S^{3}$. Let $\mathcal{C}$ be the collection of all annuli in $\partial R$, which winds at least twice algebraically in the complementary solid torus of $R$ in $S^{3}$, so for any such annulus $S$, a component of $\partial S$ is a curve on $\partial R$ of slope $(p, q)$, for some relative prime integers $p$ and $q$ with $|p| \geq 2$. Let $l_{1}$ be the core of $R$. Let $k$ be a nontrivial knot in $S^{3} \backslash l_{1}$ which has a 1-bridge presentation with respect to some annulus $S$ of the collection $\mathcal{C}$ (cf. [E, 4.3]), that is: (a) $k$ can be isotoped to intersect tranversally $\partial R$ in two points, both points lying in $S$. Then $k$ is divided into two $\operatorname{arcs} k_{1}, k_{2}$. (b) $k_{i}, i=1,2$, can be projected to $S$ to a single arc, that is, there is a disk $D_{i}$ such that $\partial D_{i}=k_{i} \cup \gamma_{i}$, where $\gamma_{i}$ is a single arc in $S$, and $\operatorname{int} D_{i} \cap \partial R=\varnothing$, int $D_{i} \cap k=\varnothing$. Let $l=l_{1} \cup l_{2}$ be a link such that $l_{1}$ is as above and $l_{2}=k$. We say that $l$ belongs to the class $\mathcal{A}$.

If $l$ is in the class $\mathcal{A}$, then $l_{2}$ has a 1 -bridge presentation with respect to some annulus $S \subset \partial R$, and it follows that $l_{2}$ can be isotoped to lie in $N(S)$. Let $T=$ $\partial N(S)$. If $l_{2}$ is not parallel to the core of $S$, then the torus $T$ is essential in $E(l)$. Note that in any case there is an essential annulus $A$ in $E(l)$, which has its boundary on $\partial E\left(l_{1}\right)$; this is as follows: Let $\partial S=s_{1} \cup s_{2}$. There are annuli $A_{1}$ and $A_{2}$ in $R$, joining the curves $s_{1}$ and $s_{2}$ to respective curves on $\partial N\left(l_{1}\right)$; let $A=(T \backslash R) \cup A_{1} \cup A_{2}$. The link $l$ admits an unknotting tunnel, which is a straight arc joining $l_{1}$ and $l_{2}$, which is disjoint from the annulus $A$.

Note that if $l \in \mathcal{A}$ and $q \geq 2$, then $l_{2}$ is a satellite knot with tunnel number one.

The following is an alternative description of the class of links $\mathcal{A}$; it follows from [E, 4.8] that these descriptions are equivalent: Let $k_{0}$ be a torus knot of type $(p, q)$ in $S^{3}$, where $|p| \geq 2$, so $k_{0}$ may be trivial. Let $L=k_{1} \cup k_{2}$ be a 2-bridge link of type $(\alpha, \beta)$ in $S^{3}$. Since $k_{2}$ is a trivial knot, there is an orientation-preserving homeomorphism

$$
f: S^{3} \backslash N^{\circ}\left(k_{2}\right) \rightarrow N\left(k_{0}\right)
$$

which takes a meridian $m_{2} \subset \partial N\left(k_{2}\right)$ of $k_{2}$ to a fiber

$$
h \subset \partial N\left(k_{0}\right)=\partial\left(S^{3} \backslash N^{\circ}\left(k_{0}\right)\right)
$$

of the Seifert fibration $D(-r / p, s / q)$ of $S^{3}$. We consider the link $l=l_{1} \cup l_{2}$, where $l_{1}$ is the core of the torus knot $k_{0}$, and $l_{2}$ is the knot $f\left(k_{1}\right)$. The link $l$ is determined by the symbol $(\alpha, \beta ; p, q)$. In Figure 8 a member of this family is shown.

(2) Let $k$ be a non-trivial $(p, q)$-torus knot or a $(p, q)$-cable of a $\left(p_{0}, q_{0}\right)$-torus knot, where $p=q p_{0} q_{0} \pm 1$; i.e. $k$ is a torus knot or a cable knot with tunnel number one (cf. [MS], [Mo], [E, 4.6]).

Let $k$ be a torus knot; then any unknotting tunnel for $k$ is isotopic to one of the tunnels $\tau_{u}, \tau_{v}, \tau_{0}$ described in [BRZ] or [MS]. There is an essential annulus $A$ in $E(k)$, which divides it into two solid tori $Q_{1}, Q_{2}$. Choose representatives of $\tau_{u}, \tau_{v}$, and $\tau_{0}$, which are arcs properly embedded in $E(k)$, are disjoint from $A, \tau_{u}\left(\tau_{v}\right)$ is contained in $Q_{1}\left(Q_{2}\right)$, and $\tau_{0}$ is contained in either of $Q_{1}$ or $Q_{2}$, for it is isotopic to an arc lying on $A$. 


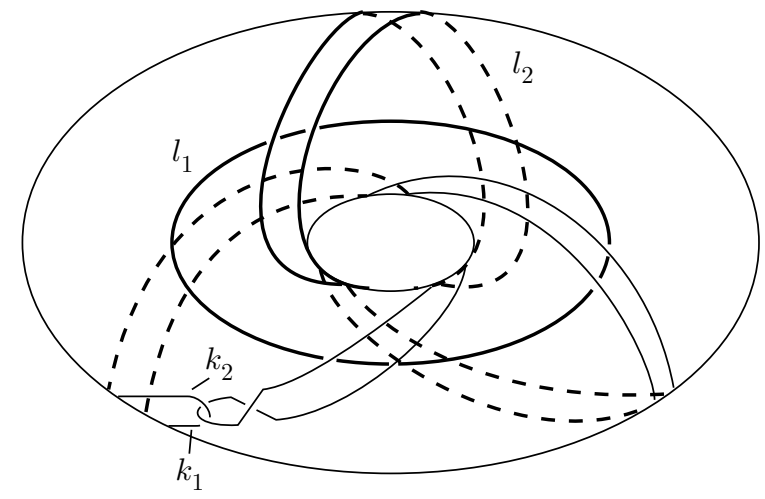

FIGURE 8

Let $k$ be a cable of a torus knot as above. Any unknotting tunnel for $k$ is isotopic to one of two unknotting tunnels described in [MS], which we denote by $\tau_{1}, \tau_{2}$. There is an essential annulus $A$ in $E(k)$, which divide it into a solid torus $Q_{1}$, and the exterior $E\left(k_{0}\right)$ of a torus knot $k_{0}$. Choose representatives of $\tau_{1}$ and $\tau_{2}$, which are arcs properly embedded in $E(k)$, are disjoint from $A$, and are (necessarily) contained in $E\left(k_{0}\right)$.

Let $k$ and $A$ be as above. $\partial A$ divides $\partial E(k)$ into two annuli; let $B$ be one of these annuli (either one if $k$ is a torus knot, and the one contained in $E\left(k_{0}\right)$ if $k$ is a cable of a torus knot $k_{0}$ ). Embed $B \times I$ into $E(k)$, where $I=[0,1]$ (and such that $A \cap(B \times I)=\partial B \times\{0\})$. Clearly

$$
\operatorname{cl}(E(k) \backslash(B \times I))=E(k) .
$$

If $\tau$ is one of the chosen tunnels of $k$, assume that its endpoints lie on $B \times\{1\}$, say these are $\left(x_{0}, 1\right)$ and $\left(x_{1}, 1\right)$, where $x_{0}$ and $x_{1}$ are distinct points in the interior of $B$. Let $k^{\prime}$ be a knot in $S^{3}-k$ divided into 2 simple arcs, $k_{1}$ and $k_{2}$, such that $k_{1}$ is an arc lying on $B \times\{1\}$ with endpoints in $x_{0}$ and $x_{1}$, and $k_{2}$ is one of the standard tunnels for $k$ described above. Define a link of two components $l=l_{1} \cup l_{2}$, where $l_{1}=k$ and $l_{2}=k^{\prime}=k_{1} \cup k_{2} . l$ is a non-simple link, for $A$ is an essential annulus in $E(l) ; l$ has an unknotting tunnel, which is the $\operatorname{arc}\left\{x_{0}\right\} \times I$. We say that $l$ belongs to the class $\mathcal{B}$.

Note that arcs on an annulus with ends on two fixed points can be parameterized by the rational fractions $\alpha / \beta$, where $\alpha$ is odd and $\beta$ is even. (Pair of $\operatorname{arcs}$ in a sphere with endpoints in four fixed points are parameterized by $Q \cup\{1 / 0\}$; such arcs are divided in three classes, depending of the points they join, and each class is parameterized by a class of fractions $\alpha / \beta$, where this class is determined by the parity of $\alpha$ and $\beta$. Arcs in an annulus with endpoints in two fixed points are in one-to-one correspondence with any of the classes of arcs of the sphere. We can choose then the class parameterized by fractions $\alpha / \beta$ where $\alpha$ is odd and $\beta$ is even.) A link $l=l_{1} \cup l_{2}$ in the class $\mathcal{B}$ can be described by a symbol $\left(p, q ; \alpha, \beta ; \tau_{i}\right)$ or a symbol $\left(p, q ; p_{0}, q_{0} ; \alpha, \beta ; \tau_{i}\right)$, depending if $l_{1}$ is a $(p, q)$-torus or a $(p, q)$-cable of a $\left(p_{0}, q_{0}\right)$-torus knot. $(\alpha, \beta)$ and $\tau_{i}$ denote the types of arcs in which $l_{2}$ is divided, where $\tau_{i}$ is one of the standard tunnels for $l_{1}$. In Figure 9 a member of $\mathcal{B}$ is shown.

Theorem 2. Let $l$ be a non-simple link with unknotting tunnel $\tau$, and $A$ an essential annulus disjoint from $\tau$. Suppose that $\partial A=a_{1} \cup a_{2}$ lie on the same component 


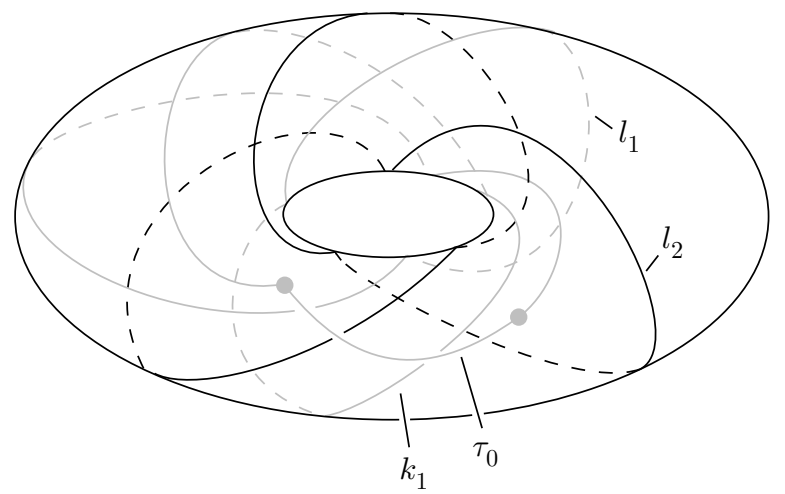

FiguRE 9

of $\partial E(l)$, say it lies on $\partial N\left(l_{1}\right)$. Then one of the following cases occurs:

(i) If $A$ is inessential in $E\left(l_{1}\right)$, then $l$ is in $\mathcal{A}$.

(ii) If $A$ is essential in $E\left(l_{1}\right)$, then $l$ is in $\mathcal{B}$.

Proof. Suppose that $\partial A \subset \partial N\left(l_{1}\right)$. A is incompressible in $M=E(l \cup \tau)$, and it is separating. $M \backslash N^{\circ}(A)$ consists of two handlebodies. $a_{1}$ cannot be a meridian of $l_{1}$, for in this case $l$ would be a non-trivial connected sum $l=k_{1} \# k_{2}$, where say, $k_{1}$ is a knot. But $E\left(k_{1}\right)$ is homeomorphic to a component of $M \backslash N^{\circ}(A)$, i.e., it is a handlebody; then $k_{1}$ is the trivial knot, which is a contradiction. Therefore $a_{1}$ is a curve of type $(p, q)$ on $\partial N\left(l_{1}\right),(p, q) \neq(1,0)$.

There are two possibilities:

Case (i). $A$ is inessential in $E\left(l_{1}\right)$. Then $A$ is parallel in $E\left(l_{1}\right)$ to an annulus $A^{\prime}$ of type $(p, q)$ in $\partial N\left(l_{1}\right) . E\left(l_{1}\right) \backslash N^{\circ}(A)$ has two components; one is a solid torus in which $l_{2}$ and $\tau$ lie, and the other is homeomorphic to $E\left(l_{1}\right)$, but this has to be a handlebody, so $E\left(l_{1}\right)$ is a solid torus and $l_{1}$ is a trivial knot. Therefore $|p| \geq 2$.

Note that $l_{1} \cup \tau$ is an unknotting tunnel for $l_{2}$, so by [E, 4.4], $l_{2}$ has a 1-bridge presentation with respect to $\partial N\left(l_{1}\right)$, and by the argument of [E, 4.7], $l_{2}$ has a 1-bridge presentation w.r.t. $A^{\prime}$. This shows that $l \in \mathcal{A}$.

Case (ii). $A$ is essential in $E\left(l_{1}\right)$. Therefore $l_{1}$ is a cable knot with tunnel number one, so $l_{1}$ is a torus knot or a $(p, q)$-cable of a $\left(p_{0}, q_{0}\right)$-torus knot, where $p=q p_{0} q_{0} \pm 1 . A$ is the standard annulus properly embedded in $E\left(l_{1}\right) . A$ divides $E\left(l_{1}\right)$ in two pieces; one is a solid torus, and the other is a manifold $N$ homeomorphic to $E(k)$, where $k$ is the companion of $l_{1}$, so $k$ is the trivial knot or a certain torus knot. We can assume that $l_{2}$ and $\tau$ lie on $N$. $A$ divides $\partial E\left(l_{1}\right)$ on two annuli, let $B$ be one of them.

Slide $l_{2}$ over $\tau$ to get an arc $\tau^{\prime}$ properly embedded in $E\left(l_{1}\right) . \tau^{\prime}$ is isotopic to one of the standard tunnels $\gamma$ for $E\left(l_{1}\right)$.

Claim. There is an isotopy carrying $\tau^{\prime}$ to $\gamma$ which keeps the annulus $A$ fixed.

The isotopy which carries $\tau^{\prime}$ to $\gamma$ transforms the annulus $A$ into an annulus $A^{\prime}$, which may intersect $A$. By looking at the intersections between the annuli and doing an innermost disk-outermost arc argument the isotopy can be deformed to one which fixes $A$, except possibly if $\gamma=\tau_{0}$ and $\tau^{\prime}$ and $\gamma$ lie on different components of $E(k) \backslash A$. But this can be fixed by assuming that if $\gamma=\tau_{0}$, then $\gamma$ and $\tau^{\prime}$ must 
lie on the same component $E(k) \backslash A$ - there is no loss of generality in doing that. If $\gamma \neq \tau_{0}$, then necessarily $\gamma$ and $\tau^{\prime}$ lie on the same side of $E(k) \backslash A$.

Let $x_{0}, x_{1}$ be the endpoints of $\gamma$. We can assume that the endpoint of $\tau$ lying on $l_{1}$ coincides with one of the endpoints of $\gamma$, say this is $x_{0}$, and that the isotopy between $\tau^{\prime}$ and $\gamma$ fixes that point. Consider an embedding of $B \times I$ into $E(k)$ so that $A \cap(B \times I)=\partial B \times\{0\}$. The isotopy carrying $\tau^{\prime}$ to $\gamma$ can be made in two steps. First, $\tau^{\prime}$ is slid over $B$ so that the complement of $B \times I$ is fixed, and keeping fixed the endpoint of $\tau^{\prime}$ lying on $x_{0}$. Second, $\tau^{\prime}$ is isotoped to $\gamma$ leaving fixed $B \times I^{\prime}$, where $I^{\prime}=[0, a]$ is a subinterval of $I . \gamma \cap(B \times I)$ consists of two straight arcs. This isotopy can be made in reverse order, in two steps. First, the endpoint of $\gamma$ on $x_{0}$ is fixed, and the other is slid over $B$, keeping fixed the complement of $B \times I$. Second, $\gamma$ is isotoped, keeping fixed $B \times I$, and finally $\tau^{\prime}$ is slid over itself to get $l_{2} \cup \tau$. This shows that $l_{2}$ can be decomposed into two arcs, $k_{1}, k_{2}$, such that $k_{1}$ is an arc in $B \times I$, consisting of a monotonic arc going from $\left(x_{1}, 1\right)$ to $\left(x_{0}, 0\right)$ union $\tau$, and $k_{2}$ is an arc in the complement of $B \times I$, which can be thought equal to $\gamma$. Note that the arc $k_{1}$ can be isotoped to a simple arc in $B \times\{1\}$, with endpoints $\left(x_{0}, 1\right)$ and $\left(x_{1}, 1\right)$. This shows that $l$ belongs to the class $\mathcal{B}$.

Note. The list of links given in Theorem 1 is not disjoint from the list of links in Theorem 2. First note that case (iv) of Theorem 1 is contained in case (iii) of the same theorem. Links satisfying case (iii) of Theorem 1 also satisfy case (i) of Theorem 2. Links satisfying case (ii) of Theorem 1 satisfy both cases of Theorem 2 . If $l_{1}$ is a $(p, q)$-cable of a $\left(p_{0}, q_{0}\right)$-torus knot, where $p=q p_{0} q_{0} \pm 1$, and if $l_{2}$ is the core of the $\left(p_{0}, q_{0}\right)$-torus knot, then $l=l_{1} \cup l_{2}$ satisfy both cases of Theorem 2 . However these are all the intersections among the different cases.

Remark. Tunnel number one knots are prime $[\mathrm{N}]$, $[\mathrm{S}]$; but it follows from Theorem 1(ii) that the same is not true for links. K. Morimoto $[\mathrm{M}]$ proved that the only composite links which admit unknotting tunnels are sums of 2-bridge knots with the Hopf link. In Theorem 1(i) we give a different proof of this fact. A third proof can be given using a stronger version of the lemma (which also follows from $[\mathrm{E}]$ ) and an argument similar to the first part of the proof of Theorem 2. A. C. Jones $[\mathrm{J}]$ proved that if a composite link has a 2-generator fundamental group, then it has two summands, one is a knot and the other is the Hopf link. The fact that a rank 2 composite link has a Hopf link summand was previously known by F. GonzálezAcuña and H. Short; see Knot surgery and primeness, Math. Proc. Cambridge Philos. Soc. 99 (1986), 89-102, page 96, line 32.

\section{REFERENCES}

[BRZ] M. Boileau, M. Rost and H. Zieschang, On Heegaard decompositions of torus knots exteriors and related Seifert fibred spaces, Math. Ann. 279 (1988), 553-581. MR 89a:57013

[E] M. Eudave-Muñoz, On non-simple 3-manifolds and 2-handle addition, Topology and its applications 55 (1994), 131-152. MR 95e:57029

[J] A. C. Jones, Composite two-generator links have a Hopf link summand, preprint.

[K] T. Kobayashi, Structure of Haken manifolds with Heegaard splittings of genus two, Osaka J. Math. 21 (1984), 437-455. MR 85k:57011

[Mo] Y. Moriah, A note on satellites and tunnel number, Kobe Journal of Math. 8 (1991), 73-79. MR 92m:57014

[M] K. Morimoto, On composite tunnel number one links, preprint.

[MS] K. Morimoto and M. Sakuma, On unknotting tunnels for knots, Math. Ann. 289 (1991), 143-167. MR 92e:57015 
[N] F. H. Norwood, Every two generator knot is prime, Proc. Amer. Math. Soc. 86 (1982), 143-147. MR 83k:57005

[S] M. Scharlemann, Tunnel number one knots satisfy the Poenaru conjecture, Topology and its applications 18 (1984), 235-258. MR 86e:57009

[T] M. Takahashi, Two-bridge knots have Property P, Memoirs Am. Math. Soc. 29 (1981). MR 82f: 57010

Instituto de Matematicas, U.N.A.M. Circuito Exterior, Ciudad Universitaria, 04510 Mexico D. F. Mexico

E-mail address: eudave@servidor.unam.mx

Current address: Department of Mathematics, University of Texas, Austin, Texas 78712

Department of Mathematics, Kobe University, Rokko, Kobe 657, Japan

E-mail address: uchida@math.s.kobe-u.ac.jp

Current address: Department of Mathematical Sciences, Yamagata University, Yamagata 990, Japan

E-mail address: yuchida@kszaoh3.kj.yamagata-u.ac.jp 